\title{
COMPORTAMIENTO FRENTE AL FUEGO DE TABLEROS Y MADERA DE PINO RADIATA CON Y SIN PINTURA RETARDANTE DE LLAMA
}

\section{FIRE PERFORMANCE OF BOARDS AND PINE RADIATA WOOD WITH AND WITHOUT FLAME RETARDANT PAINT}

R. Garay ${ }^{1}$, M. Henriquez,

\begin{abstract}
RESUMEN
En aplicaciones para la construcción se requiere aportar al conocimiento del comportamiento de la madera y tableros a base de madera frente a la acción del fuego para mejorar condiciones de uso en servicio. Se comparó en tableros a base de madera y en madera sólida de pino radiata el efecto de una pintura retardante de fuego y testigos sin pintar, con el propósito de discriminar entre tipos de tableros y comparar con respecto a la madera sólida.

Los tableros ensayados fueron: Contrachapado estructurales de Pino radiata D. Don, Oriented Strand Board (OSB), Medium Density Fiberboard (MDF), Hard Board (HB) y tableros de partículas, los que fueron pintados superficialmente con pintura retardante de llama y comparados con tableros sin protección. Se incluyó en el estudio, madera de pino radiata con y sin retardante de llama.

El análisis estadístico indicó diferencias significativas en pérdida de peso e índice de carbonización para todos los tableros con retardante de llama al compararlos con los tableros sin protección. Entre tableros estructurales: contrachapados y OSB, el contrachapado con retardante de llama fue el que presentó un mejor comportamiento frente al fuego y, HB presentó el comportamiento más deficiente.
\end{abstract}

Se demostró que una pintura retardante de llama resulta efectiva en la protección de los tableros estudiados al comparar con tableros sin protección.

La aplicación de esta norma de ensayo fue un buen indicador del comportamiento al fuego y aportó información significativa de los tableros, aconsejándose su empleo como un complemento de los ensayos de resistencia al fuego que se realizan a escala real.

Palabras claves: Tableros a base de madera, pintura retardante de llama, comportamiento al fuego, pérdida de peso, índice de carbonización.

\section{ABSTRACT}

In housing contruction to require increase the knowledge of these materials behavior against to deterioration agents. The security opposite to the fire action is fundamental to improve their use in service.

The boards tested were: radiata pine structural Plywood, Oriented Strand Board (OSB), Medium Density Fiberboard (MDF), Hard Board (HB) and particleboard, which were protected with

\footnotetext{
${ }^{1}$ Departamento de Ingeniería de la Madera, Facultad de Ciencias Forestales, Universidad de Chile. Santiago- Chile

Autor para correspondencia : rgaray@uchile.cl

Recibido: 05.08.2009 Aceptado: 30.12.2009
} 
flame retardant paint and were compared with boards without protection. Radiata pine wood with and without flame retardant paint was included in the study.

The statistical analysis showed significant differences in weight loss and carbonization index for all the boards with flame retardant compared them with the boards without protection. Between structural boards: plywood and OSB, plywood with flame retardant was the one that presented best fire performance, while HB presented the worst behavior.

It was demonstrated that the flame retardant paint of turns out to be effective in the protection of the boards studied on having compared with boards without protection.

The implementation of this standard test was a good indicator of the fire performance and contributed with significant information of the boards, its utilization is advised as a complement to fire resistance test carried out at real scale.

Keywords: wood board, flame retardant paint, fire performance, loss of weight, carbonization index.

\section{INTRODUCCIÓN}

El Comportamiento frente al fuego en materiales de construcción se evalúa a través de parámetros tales como tiempo de ignición, pérdida de peso, índice de carbonización, propagación de llama, tasa de calor liberado y generación de humos, entre otros. La resistencia al fuego se mide en minutos y es la capacidad que exhibe un elemento de construcción para conservar durante un periodo determinado de tiempo sus cualidades estructurales dentro de ciertos límites de temperatura. En base a este último concepto están definidas las exigencias de resistencia al fuego, condiciones de seguridad contra incendios, normativas de resistencia al fuego de los materiales para la construcción, por La Ordenanza General de Urbanismo y Construcción (OGUC) del Ministerio de Vivienda y Urbanismo. Las clases de resistencia al fuego van desde F15 a F180, indicando esta codificación la cantidad de minutos que las estructuras deben resistir (MINVU 2004).

OGUC define la Protección Pasiva como: "la que se basa en elementos de construcción que por sus condiciones físicas aíslan la estructura de un edificio de los efectos del fuego durante un determinado lapso de tiempo, retardando su acción y permitiendo en esa forma la evacuación de sus ocupantes antes del eventual colapso de la estructura y dando, además, tiempo para la llegada y acción de bomberos". Los elementos de construcción o sus revestimientos pueden ser de materiales no combustibles, con capacidad propia de aislación o por efecto intumescente o sublimante frente a la acción del fuego (MINVU 2004).

La norma Chilena NCh 935/1-of.97, establece el procedimiento para determinar la resistencia al fuego de las pinturas intumescentes válido sólo para estructuras metálicas y no para la madera, dicha norma no señala valores exigidos, que son inherentes a cada pintura y depende de los espesores aplicados sobre una estructura de acero de una masividad determinada (Infante 2008). Las pinturas intumescentes, utilizadas sobre metales, están certificadas en IDIEM bajo el protocolo $\mathrm{N}^{\circ}$ 238.148-238.378.

En madera, la resistencia al fuego se logra con grandes escuadrías o revistiéndola con productos minerales, como las placas de yeso-cartón o fibrocemento. Respecto a barnices ignífugos y pinturas retardantes del fuego, OGUC no señala exigencias específicas en particular; salvo establecer el uso de la norma chilena NCh 1974.of. 86, relativa a prevención de incendio en edificios y determinación del retardo al fuego. Por este motivo sólo hay formas de medir sus características, pero no su obligatoriedad. 
La norma NCh 1974.of. 86, permite determinar de modo cuantitativo las propiedades retardantes de llama producidas por una o varias capas de pintura aplicadas sobre superficies de madera a través de la determinación de pérdida de masa e índice de carbonización de las probetas recubiertas con dicha pintura. Este método se utiliza solamente para medir y describir las propiedades de las pinturas en respuesta a la llama, bajo condiciones controladas de laboratorio. Por lo tanto no debe ser usado para la descripción, evaluación o reglamentación del riesgo de incendio real.

Los productos de madera y tableros, son materiales que presentan numerosas ventajas como materiales de construcción, tales como: excelentes características de habitabilidad y resistencia sísmica, calidez, rapidez de construcción, facilidad de transporte, buena aislación térmica y acústica y menor costo. Sin embargo, aún son considerados como materiales ligeros y temporales en las viviendas, prefiriéndose los materiales tradicionales como albañilería y hormigón (Forest Products Laboratory 1999)

Hace muchos años en los países desarrollados con alto ingreso per-capita y más recientemente en los países en vías de desarrollo como Chile, se ha optado por sistemas constructivos basados en estructuras de madera o de metal, revestidos exteriormente por tableros estructurales (Neira 2001).

Dentro de estos sistemas constructivos, los tableros estructurales contrachapados y OSB (Oriented Strand Board), son altamente utilizados para la construcción de revestimientos como: soportes de cubiertas y diafragma, forjados, cerramiento de fachadas, tabiques divisorios, techos, pisos e incluso en la fabricación de muebles (Garay et al. 2009, Peraza 1998). Estos materiales son utilizados por empresas constructoras que han incorporado alternativas distintas al cemento, ladrillos o bloques en segundos pisos y también para la construcción de viviendas sociales, por sus características de excelente confort térmico (Neira 2001).

Sin embargo y pese a su amplia utilización, existe desconfianza del comportamiento de las estructuras de madera y tableros frente a la acción del fuego, ya que persiste el temor frente a un incendio (Ramírez y Di Pace 2002). Esto no debe ser un impedimento para su empleo en la construcción de viviendas. Los productos de madera, en comparación con los materiales habitualmente utilizados, pueden llegar a presentar incluso una mayor seguridad frente a otros materiales incombustibles, que pierden su estabilidad estructural cuando son sometidos a determinadas temperaturas (Garay et al. 2009).

Un ejemplo de estructuras resistentes al fuego es sugerido por Lp Chile (2008) en cuya composición considera un tabique interno de pino radiata en escuadría de $2 \times 3$ " cuya cara exterior posee un tablero de fibrocemento de $15 \mathrm{~mm}$ de espesor, mientras que la cara interna tiene un tablero OSB de $15 \mathrm{~mm}$ de espesor. Esta estructura al ser ensayada para medir su resistencia al fuego a escala real resiste un tiempo de 30 minutos antes de colapsar, lo que según la norma NCh 935 implica un índice F30.

Bajo ciertas condiciones la madera presenta una buena resistencia al fuego gracias a la formación de una capa carbonizada; ya que expuesta a altas temperaturas se descompone para proporcionar una capa aislante de carbón que retarda aún más la degradación de la madera. La capacidad de resistencia al fuego de una estructura de madera depende de su escuadría, así, la cantidad de carbonización de la sección transversal es el principal factor en la resistencia al fuego de miembros estructurales de madera. En cambio en otras aplicaciones, su comportamiento frente al fuego es limitado puesto que cuando forma parte de muebles, revestimientos de muros y cielos u otros usos que emplean piezas más delgadas, arde con facilidad (Forest Products Laboratory 1999).

Los ensayos de reacción al fuego suelen realizarse en un cono calorímetro (Harada et al, 2003), las mediciones de tasa de calor liberado se realizan de acuerdo a la norma ISO 5660 y el humo específico de la zona de extinción se evalúa de acuerdo con la norma ASTM E 1354-92. El flujo de calor incidente normalmente es de $50 \mathrm{~kW} / \mathrm{m}^{2}$ en probetas de $100 \mathrm{~mm}$ x $100 \mathrm{~mm}$. INFOR (1999) encargó un ensayo de reacción al fuego al laboratorio Sueco $\mathrm{AB}$ Trätek con el propósito de probar varios productos 
retardantes de llama aplicados por impregnación en la madera. Bajo condiciones de ensayo a escala real en madera sin tratar, la pérdida de peso fue de $81,4 \%$, mientras que en la madera impregnada con los productos retardantes de llama, la pérdida de peso después del ensayo fluctuó entre 75,2 y 58,4\%; por su parte, en tablero de yeso cartón el porcentaje de pérdida de peso fue de $14,9 \%$, demostrando su mejor reacción frente al fuego.

Los ensayos de resistencia al fuego utilizan métodos de combustión a escala real y determinan el tiempo de duración de una estructura específica, tal como un tabique corta fuego, construido de diferentes materiales, espesores y composiciones, pudiendo o no ser considerado el empleo de productos retardantes de llama, como pinturas, ya que éstas sólo representan una parte más de la composición total de la estructura (Garay y Ahumada 2008).

Las pinturas retardantes de llama, permiten que la madera y tableros puedan resistir periodos más largos de tiempo en exposición a fuentes directas de fuego, éstas mejoran las propiedades ignifugas de estos materiales, aumentando su punto de ignición y disminuyendo el desplazamiento y penetración de la llama, además disminuyen la densidad de sustancias volátiles y el exceso del calor del medio (Levow and Winandy 1998). El retardo al fuego es la capacidad de una pintura para retardar la propagación de la llama sobre un substrato.

La mayoría de las formulaciones para mejorar el comportamiento de la madera frente al fuego incluyen productos químicos basados en el fósforo, nitrógeno, boro, silício y en otras combinaciones que producen sinergias con los anteriores (Decorespacio 2008).

Existe una gran variedad de compuestos químicos retardantes de fuego usados en madera y tableros en base a madera, tanto por impregnación, incorporación a la masa o aplicación superficial (Forest Products Laboratory 1999).

Su formulación depende de muchos factores, entre ellos las características propias del sustrato, la presencia de aditivos en el material, el proceso de elaboración del producto en relación a variables y riesgos implícitos del proceso y las condiciones de uso del material al que será expuesto, debiendo decidir en base a estos factores, la incorporación a la masa de fibras en tableros ó la aplicación de un producto superficial tipo pintura (Garay et al. 1996).

Al realizar una evaluación técnica de incorporación de retardantes de llama en base a polifosfatos de amonio en tableros de densidad media Garay et al. (1996), encontraron que estos productos resultaban efectivos en la disminución superficial de llama, según especificaciones de la norma ASTM D 3806- 90a.

Jun-wei et al. 2007 analizaron la formulación de un recubrimiento pirorretardante preparado con resina de poliéster insaturado y resina epóxica de dos componentes como matriz de resinas y polifosfato de amonio (APP) como fuente ácida, melamina (Mel) como agente espumante y pentaeritritol (PER) como agente de carbono, grafito expandible como agente sinérgico, con adición de dióxido de titanio $\left(\mathrm{TiO}_{2}\right)$, disolventes y otros aditivos. Los resultados mostraron que había excelentes propiedades físico-químicas de la capa. Cuando el espesor del recubrimiento en la madera fue 2,0 mm, el límite de resistencia al fuego llegó a 210 min. Mediante diversos sistemas de detección de imagen se estudió los mecanismos de acción físico en la descomposición y el rol de cada compuesto químico durante la combustión, estableciendo la relevancia de encontrar buenas mezclas entre la resina base de la pintura y los aditivos piroretardantes que actúan formando una capa carbonizada resistente para retardar la llama.

Cuando la madera ha sido sometida a fuentes directas de fuego, puede llegar a perder aproximadamente entre 0.5 y $1 \mathrm{~mm}$ de material por minuto de exposición, dependiendo del tipo de madera, lo que es ocasionado por el fenómeno de carbonización. Cuando la madera es pre-tratada con 
alguna sustancia retardante de fuego, puede resistir un período de tiempo mayor, lo cual no significa que la madera no sufrirá carbonización (Arquicity 2006).

Cuando los retardantes son aplicados sobre los sustratos, éstos son absorbidos y eliminan el espacio para el oxígeno con objeto de detener el fuego y su propagación. Cuando la fuente del fuego continúa en contacto con el material impregnado por el retardante, el objeto se consume de forma mucho más lenta a lo habitual, lo que permite extinguir el incendio (QuimiNet 2006).

Hashim et al (2009) incorporaron retardantes de llama en base a aluminato de sodio, borato de zinc y aluminio trihidrato a la masa de fibras para tablero de fibras de densidad media (MDF). Los que fueron evaluados mediante el uso de la norma ASTM D 1360-90 (1990) Los resultados mostraron que el índice de carbonización y la pérdida de peso se redujeron con todos los retardantes evaluados, el hinchamiento en espesor y la absorción de agua de los tableros disminuyeron. El módulo de ruptura (MOR) no se vio afectado por los tratamientos retardantes de llama y la tracción interna (IB) mostró una pequeña reducción en comparación con los testigos. Aluminato sódico mostró el mejor desempeño en la reducción de la degradación térmica seguido de trihidrato de aluminio y el borato de zinc.

En una caracterización de pisos laminados (Garay y Ahumada 2008) fabricados en base a tableros del tipo High Density Fiberboard (HDF), se estableció que los pisos tienen un comportamiento frente al fuego deficiente al ser evaluados bajo la norma ASTM D1360-90a (1994), determinándose pérdidas de peso de 3,04 y 2,94\% para ambos tipos estudiados (las principales diferencias entre ambos eran el precio, menor y mayor valor y la veta de imitación madera que presentaba en la superfcie. Los pisos laminados presentaron una pérdida de peso mayor al de molduras MDF que habían sido estudiadas anteriormente, con un mayor daño concentrado de carbonización en la zona ensayada, aunque no traspasa el tablero, debido a su estructura interna y alta densidad. Lo más interesante de observar fue que la cubierta de barniz de alta resistencia al tráfico (overlay) no actúo inhibiendo la combustión, por el contrario participó en el proceso y se quemó de forma importante, más que en molduras con imprimante que habían sido evaluadas anteriormente (Garay 2003).

En el estudio de Chuen-Shii et al. (2009), se investigó un nuevo retardador de llama para formular un revestimiento ignífugo intumescente (FICR). Se emplearon distintas proporciones de polvo de grafito artificial (POP), sericita $\left(\mathrm{Al}_{4}(\mathrm{OH})_{4}\left(\mathrm{KAl}-\mathrm{Si}_{3} \mathrm{O}_{10}\right)_{2}\right)$ y mezcla de grafito/sericita. FICR consistió en 19,8\% de retardador de llama, 15\% de agente de deshidratación, 18\% de agente espumante, $7,2 \%$ de resina base y $40 \%$ de disolvente, el que fue preparado y aplicado en la parte superior de un contrachapado. Se estableció qué con porcentaje de sericita superior al $75 \%$ se consigue pasar la exigencia de retardo exigida y que en ese caso no es necesario incorporar polvo de grafito artificial: Lo más importante fue haber encontrado una alternativa de retardador de llama a base de compuestos naturales (sericita), que puede ser obtenido a través de un proceso simple y convencional de la minería y no requiere agente de carbono.

Investigar los compuestos retardantes de llama, comprender los conceptos de resistencia al fuego, conocer las exigencias impuestas por OGUC, delimitar adecuadamente donde deben ser utilizadas las estructuras y los diversos tipos de materiales, permite dimensionar de manera más adecuada la relevancia de cada solución constructiva y entregar respuestas respecto a las reales posibilidades de la madera y sus derivados en el campo de la construcción.

El objetivo de esta investigación fue comparar el comportamiento al fuego de diferentes tipos de tableros comerciales a base de madera con y sin la aplicación de una pintura retardante de llama frente a la acción de una cantidad exacta de $5 \mathrm{ml}$ de combustible. 


\section{MATERIALES Y MÉTODOS}

En la tabla 1 se resumen las características más relevantes del material de ensayo utilizado.

Tabla 1. Tipo de sustrato y sus características

\begin{tabular}{lccc}
\hline Tipo de Sustrato & $\begin{array}{c}\text { Densidad } \\
\left(\mathrm{kg} / \mathrm{m}^{3}\right)\end{array}$ & $\begin{array}{c}\text { Contenido Humedad } \\
(\%)\end{array}$ & $\begin{array}{c}\text { Dimensiones } \\
(\mathrm{mm})\end{array}$ \\
\hline MDF & 520 & 11 & $300 \times 135 \times 15$ \\
\hline OSB & 560 & 8 & $300 \times 135 \times 15$ \\
\hline Contrachapados & 560 & 8 & $300 \times 135 \times 15$ \\
\hline Partículas & 593 & 10 & $300 \times 135 \times 15$ \\
\hline HB radiata & 1000 & 7 & $305 \times 150 \times 32 *$ \\
\hline Pino ran & 445 & 11,7 & $300 \times 135 \times 15$ \\
\hline
\end{tabular}

* Debe tenerse en cuenta que este tablero posee un espesor comercial de 3,2 mm, no existe en $15 \mathrm{~mm}$, lo que habría sido más adecuado para fines comparativos.

Se utilizó un retardante de llama, marca Ceresita (CERSAX-400) Esmalte industrial brillante de alta resistencia química, formulado a base de resinas caucho clorado sin contenido de metileno y pigmentos con resistencia a ambientes industriales agresivos. Como integrante de un sistema caucho clorado presenta una excelente resistencia a la corrosión, en ambientes industriales y marinos. De rápido secado y puede aplicarse en condiciones de elevada humedad y baja temperatura. Tiene buena retención de brillo expuesto a luz solar. La pintura fue aplicada sobre la madera de pino radiata y sobre los tableros,

Los equipos e instrumentos utilizados en este estudio fueron una cabina o cámara de combustión hermética, la que en su interior debe mantener condiciones controladas de humedad relativa (50+-5\%), temperatura $\left(23+-2^{\circ} \mathrm{C}\right)$ y velocidad del aire comprendida entre 0,05 y $0,2 \mathrm{~m} / \mathrm{s}$; un soporte metálico para sostener la probeta; un recipiente de bronce cuyas dimensiones son $24 \mathrm{~mm}$ de diámetro exterior, $17 \mathrm{~mm}$ de altura exterior, $1 \mathrm{~mm}$ de espesor de pared, $6 \mathrm{ml}$ de capacidad total para una cantidad exacta de $5 \mathrm{ml}$ de combustible; balanza analítica de $0,01 \mathrm{~g}$ de precisión y pie de metro de $0,01 \mathrm{~mm}$ de precisión.

El método de ensayo se describe en la norma ASTM 1994. American Society for Testing Materials. D1360-90a. Standard Test Method for Fire Retardancy of Paints (Cabinet Method), equivalente a la norma NCh.1974.of. 86. "Prevención de incendios. Pinturas. Determinación de retardo al fuego". Las que especifican los siguientes aspectos:

Se requiere que las probetas sean acondicionadas por 15 días antes de aplicar la pintura (50+-5\% de humedad relativa y $23+-2^{\circ} \mathrm{C}$ de temperatura). La pintura se aplica con brocha uniformemente con un espesor de película de $27 \mu \mathrm{m}$, se debe dejar secar la primera capa 24 horas respecto a la segunda. Se acondicionaron por 14 días antes de realizar el ensayo.

Se consideraron 10 repeticiones para cada tipo de sustrato con y sin retardante de llama. Se midió para todas las probetas peso y espesor inicial, este último se midió en un punto que equidista a $10 \mathrm{~cm}$. del centro de la base de la probeta. Luego las probetas se colocaron con una inclinación de $45^{\circ}$ en la cámara de combustión descrita y fueron sometidas a una llama normalizada originada por una cantidad fija de $5 \mathrm{ml}$ de alcohol etílico absoluto, cuyo punto de ebullición es de $78,5^{\circ} \mathrm{C}$, la combustión prosiguió hasta que el alcohol se consumió completamente.

Posteriormente se determinó en una balanza la pérdida de peso para cada probeta ensayada, por medio de las siguientes formulas:

Pérdida de peso: $P_{P}=P_{I}-P_{F}$

Donde $\mathrm{P}_{\mathrm{p}}=$ pérdida de peso; $\mathrm{P}_{\mathrm{I}}=$ peso inicial; $\mathrm{P}_{\mathrm{F}}=$ peso final. Unidades en $\mathrm{g}$. 
El índice de carbonización se determina partiendo la probeta al finalizar el ensayo por el centro y en el sentido longitudinal con una sierra circular de carpintería. Utilizando un pie de metro se mide el espesor carbonizado, largo y ancho máximo de carbonización y se calcula mediante la fórmula siguiente:

Índice de carbonización (INC \%): INC $(\%)=\frac{l_{M A X} * a_{M A X} * e_{C}}{V_{\text {INICIAL }}} * 100$

Donde $1_{\max }=$ largo máximo de carbonización superficial; $\mathrm{a}_{\max }=$ ancho máximo de carbonización superficial; $\mathrm{e}_{\mathrm{c}}=$ espesor carbonizado (diferencia entre espesor inicial y el espesor no carbonizado) y $\mathrm{V}_{\text {INICIAL }}=$ Volumen inicial de la probeta. Dimensiones en $\mathrm{mm}$ y volumen en $\mathrm{mm}^{3}$.

De acuerdo a lo establecido en la norma NCh 1974, se calcula el valor promedio y desviación estándar para la pérdida de peso y para el índice de carbonización.

El diseño estadístico consideró un análisis bifactorial para los distintos tipos de tableros con y sin retardante de llama. Mediante análisis de varianza para los factores tipo de sustrato y tipo de protección (con y sin retardante de llama), se establece la significancia estadística de los factores a través de las variables respuesta pérdida de peso e índice de carbonización .

Se realizó una prueba de contrastes para establecer qué tipo de sustratos presentaron diferencias significativas.

\section{RESULTADOS Y DISCUSIÓN}

En las Figuras 1 y 2 se presentan imágenes que comparan la apariencia que adquirieron los tableros una vez finalizado el ensayo.

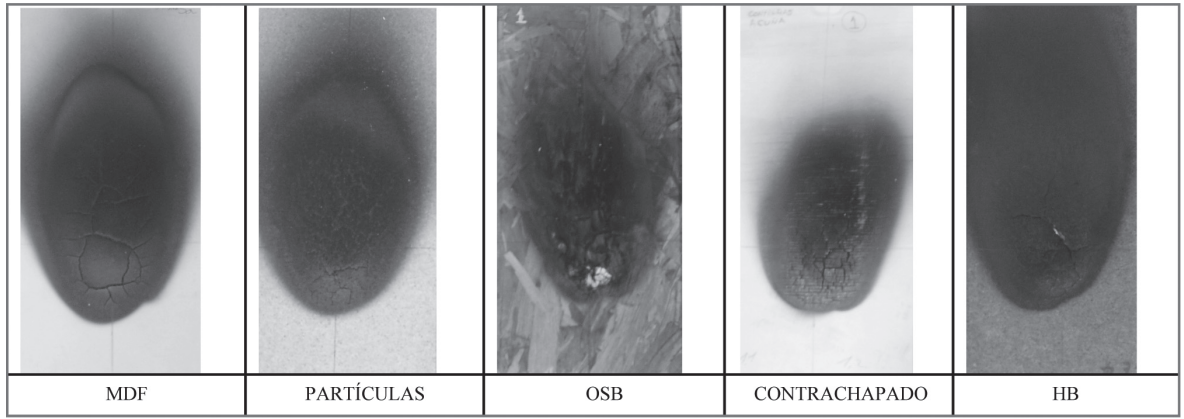

Figura 1. Aspecto de los tableros sin retardante de fuego después del ensayo.

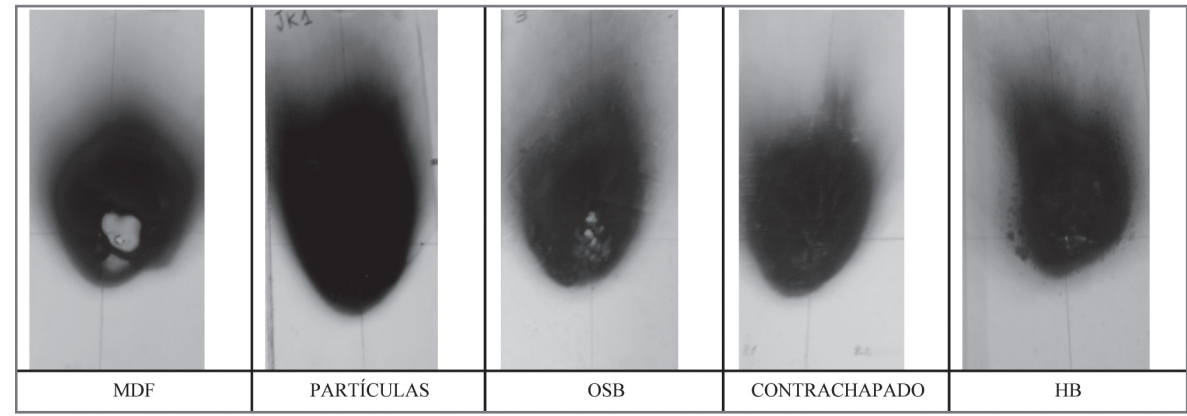

Figura 2. Aspecto de los tableros con retardante de fuego después del ensayo. 
En la Tabla 2 se informa, según lo estipulado en la norma ASTM D1360-90a (1994), pérdida de peso, tiempo de duración del ensayo e índice de carbonización (INC) de los sustratos ensayados.

Tabla 2. Comportamiento frente al fuego de sustratos ensayados

\begin{tabular}{|c|c|c|c|}
\hline Tipo de Sustrato & Pérdida de peso $(\%)$ & $\begin{array}{l}\text { Tiempo de duración del ensayo } \\
\text { (seg)/desviación estándar }\end{array}$ & $\begin{array}{l}\text { Índice de Carbonización } \\
(\%) / \text { desviaciónestándar }\end{array}$ \\
\hline MDF s/R & 5,16 & $488,76 / 64,16$ & $19,46 / 5,4$ \\
\hline MDF c/R & 3,24 & $430,68 / 365$ & $7,7 /_{10}$ \\
\hline Partícula s/R & 3,04 & $479,34 / 61,24$ & $8 / 1,0$ \\
\hline Partícula c/R & 1,92 & $473,12 / 22.71$ & $4,96 / 0,7$ \\
\hline $\mathrm{OSB} \mathrm{s} / \mathrm{R}$ & 3,22 & $577,80 / 195,49$ & $13,64 / 7,7$ \\
\hline $\mathrm{OSB} c / \mathrm{R}$ & 2,52 & $564,20 /_{86,34}$ & $11,82 / 2,3$ \\
\hline Contrachapado $\mathrm{s} / \mathrm{R}$ & 2,46 & $444,20 / 52,13$ & $11,34 / 2,2$ \\
\hline Contrachapado c/R & 1,02 & $437,80 / 23,69$ & $5,46 / 2,3$ \\
\hline $\mathrm{HB} \mathrm{s} / \mathrm{R}$ & 19,08 & $470,32 / /_{17,37}$ & $72,9 / 1,4$ \\
\hline $\mathrm{HB} \mathrm{c} / \mathrm{R}$ & 4,86 & $525,42 / 3201$ & $31,62 / 57$ \\
\hline Pino radiata s/R & 5,01 & $466,2 /_{27,4}$ & $11,56 / 2,86$ \\
\hline Pino radiata $\mathrm{c} / \mathrm{R}$ & 2,55 & $492,5 / 32,6$ & $7,25 / 3,20$ \\
\hline
\end{tabular}

$\mathrm{c} / \mathrm{R}=$ con retardante de llama

$\mathrm{S} / \mathrm{R}=$ sin retardante de llama

De la Tabla 2, se observa que para todos los tableros y para la madera de pino radiata, la pérdida de peso e índice de carbonización fueron más altos en sustratos sin retardante de llama al comparar con sustratos con retardante de llamas.

Los tiempos de ensayo son sólo referenciales, no son equivalentes a los tiempos de resistencia al fuego, los que dan origen a los códigos F15 a F180 determinados en ensayos a escala real, en donde la fuente calórica es significativamente mayor que la entregada en este ensayo por la combustión de $5 \mathrm{ml}$ de etanol.

Un aspecto metodológico que desincentiva a sacar deducciones en base al tiempo de duración del ensayo, se relaciona con que parte del tiempo de duración del ensayo es gastado en calentar el dispositivo de bronce que contiene el etanol, de esta manera, cuando el ensayo se inició con el dispositivo frío, el ensayo fue más largo que aquellos en los que sólo se repuso el etanol y se continuó con el ensayo siguiente. Por lo anterior, la duración del ensayo no evalúa estrictamente el comportamiento al fuego del sistema sustratoprotector, sino que incluye tiempos inherentes al método el que puede variar, según lo comentado. Este aspecto no se señala de manera explicita en la norma, por lo que se recomienda contemplar un calentamiento previo del dispositivo de bronce antes de ensayar, de manera de igualar las condiciones de ensayo.

En tableros contrachapados se observó una disminución tanto en pérdida de peso como en índice de carbonización obteniéndose los mejores resultados, como se observa en la Figura 3.

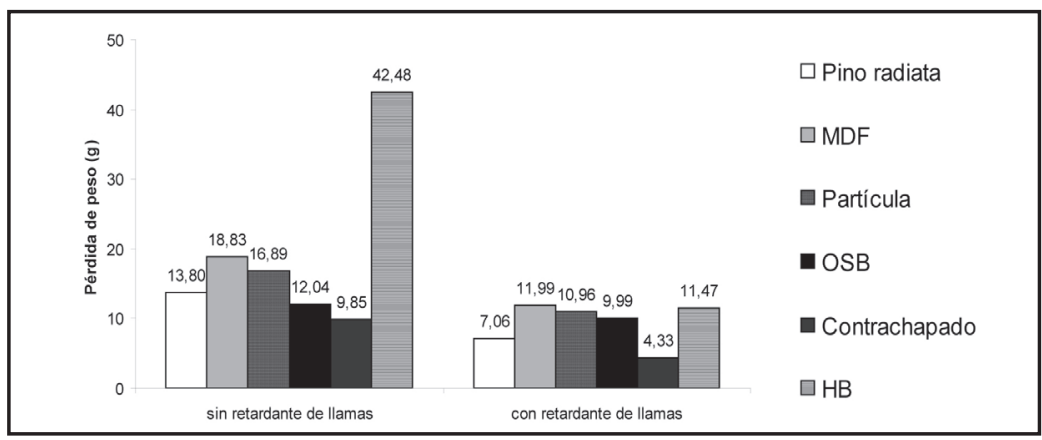

Figura 3. Comparación en pérdida de peso de los sustratos ensayados. 
En tablero HB sin retardante de llamas se tuvo un comportamiento deficiente. Considerando que son materiales no estructurales, que sus aplicaciones rara vez son en superficies amplias y son más comúnmente utilizados en fondos de cajones y en puertas de bajo costo, es que se considera en esta aplicación mayor peligro sin resguardos adecuados. Se observó que al aplicar retardante de llamas disminuyó significativamente la pérdida de peso. Debido a su bajo espesor $(3,2 \mathrm{~mm})$ este tablero es más rápidamente atravesado por la llama durante la combustión y representa un peligro mayor frente al comportamiento al fuego.

En tableros OSB hubo una incidencia más baja del retardante de llama, el cual presentó una baja diferencia en la pérdida de peso al comparar entre tablero con retardante de llama y tablero sin retardante de llama.

El tablero contrachapado presentó el valor más bajo en pérdida de peso $(1,02 \%)$, mientras que en OSB este valor es de 2,52 \%. Esta comparación es relevante ya que ambos tableros compiten por aplicaciones similares como tableros estructurales. Tanto la composición interna de estos tableros, como su mayor densidad actúan de mejor forma para repeler la llama,

Los tableros MDF y de partículas tienden a ser empleados en aplicaciones similares, como muebles, carpintería de terminación, closet y estanterías en general, por este motivo observar el comportamiento entre ambos resulta interesante, inicialmente la pérdida de peso en MDF es mayor $(5,16 \%)$ que en partículas $(3,04 \%)$, sin embargo al aplicar un retardante de llama, la pérdida de peso tiende a ser más baja y muy similar.

Finalmente, se destaca que al comparar los tableros, excepto HB con la madera sólida de pino, todos ellos tienen un mejor comportamiento frente al fuego cuando están protegidos con un retardante de llama y su pérdida de peso es menor.

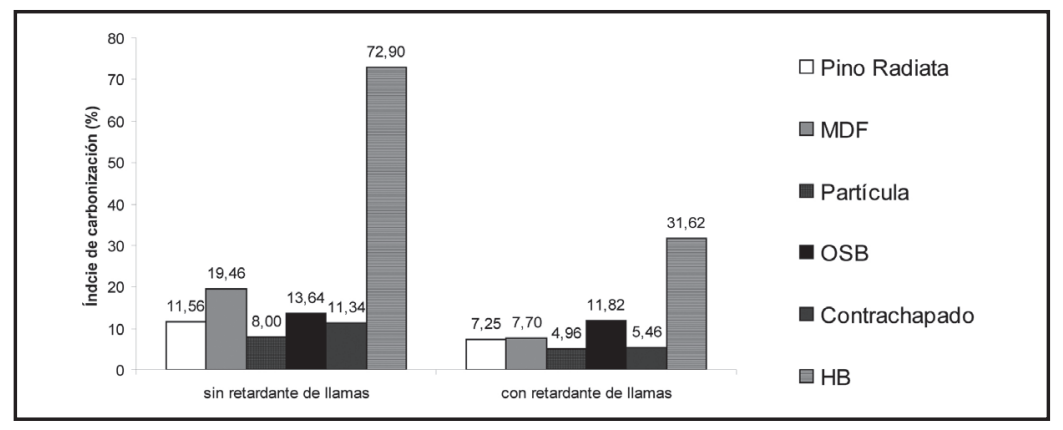

Figura 4. Comparación del índice de carbonización

En la Figura 4, se presenta el índice de carbonización de los sustratos ensayados, éste muestra una comparación del volumen carbonizado en relación al volumen total de la probeta, el que puede ser comparado visualmente en la Figura 1 y 2.

El tablero más afectado fue HB sin retardante, con un $72,9 \%$ de su volumen carbonizado, la llama atravesó el tablero en el espesor. La mayor densidad de este tablero no influyó en su comportamiento al fuego, fue más relevante el bajo espesor y la uniformidad de su estructura interna.

El tablero MDF entregó un valor de INC de 19,46\% sin retardante de llama, lo que significa que alrededor de un $20 \%$ de su volumen se carbonizó y el INC bajó a 7,7\% cuando se aplicó un retardante de llama.

Valores más bajos de INC se observaron en tableros de partículas (8 y 4,96\% con y sin retardante respectivamente), la llama no se propaga significativamente en volumen en estos tableros, 
el calor focalizado provocó una pérdida de peso menor a lo esperado, lo que se explica por la mayor densidad superficial de este tablero.

Los tableros contrachapados con retardante de llama logran el mejor desempeño en cuanto a índice de carbonización, en este caso el plano de adhesivo actúa bloqueando el avance de la llama, consumiendo en esta zona parte importante de la energía calórica liberada por la combustión del alcohol.

La madera de pino radiata con retardante, también presenta un buen comportamiento en cuanto al índice de carbonización, el daño se produjo en forma superficial más que en profundidad, lo que incide en el valor final de índice de carbonización

Se determinó que existe diferencia estadísticamente significativa, con 95\% de confianza, para ambas variables respuesta (pérdida de peso e índice de carbonización). Por medio de análisis de contraste múltiple se corroboró que los tableros contrachapados con retardante de llama tuvieron menor pérdida de peso e índice de carbonización comparado con los otros sustratos, además se pudo establecer que la presencia de retardante implicó siempre menor pérdida de peso e índice de carbonización al comparar con tableros sin retardante de llama. Un detalle del análisis estadístico efectuado se presenta a continuación:

Para tableros con y sin retardante de llamas, se realizó un análisis de varianza para los factores tipo de sustrato con y sin retardante de llamas y pérdida de peso (Tabla 3) para comprobar si existen diferencias significativas entre las medias de los factores.

Tabla 3. Análisis de varianza para pérdida de peso en tableros de madera.

\begin{tabular}{cccccc}
\hline \multicolumn{6}{c}{ Análisis de la Varianza } \\
\hline Fuente & Sumas de cuadrados & Gl. & Cuadrado Medio & Cociente-F & P-Valor \\
\hline Entre factores & 258,26 & 1 & 258,26 & 14,67 & 0,0005 \\
\hline $\begin{array}{c}\text { Dentro de los } \\
\text { factores }\end{array}$ & 669,17 & 38 & 17,60 & & \\
\hline Total (Corr.) & 927,44 & 39 & & & \\
\hline
\end{tabular}

Se determinó que existen diferencia estadísticamente significativa, con un 95\% de confianza, entre la pérdida de peso entre los tableros de madera recubiertos con el protector retardante de llama (2) y los tableros sin recubrimiento (1). Por medio de un análisis de contraste múltiple (Figura 5) se corroboró que los tableros sin protector perdieron mayor peso que los que estaban recubiertos por el producto retardante de llamas.

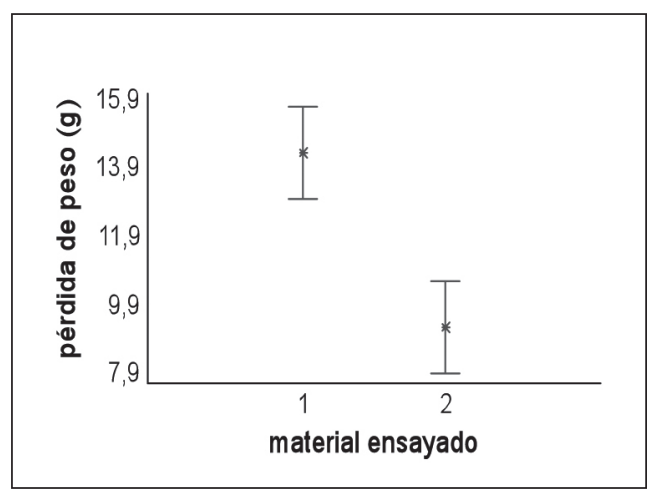

Figura 5. Pérdida de peso entre tableros con y sin retardante

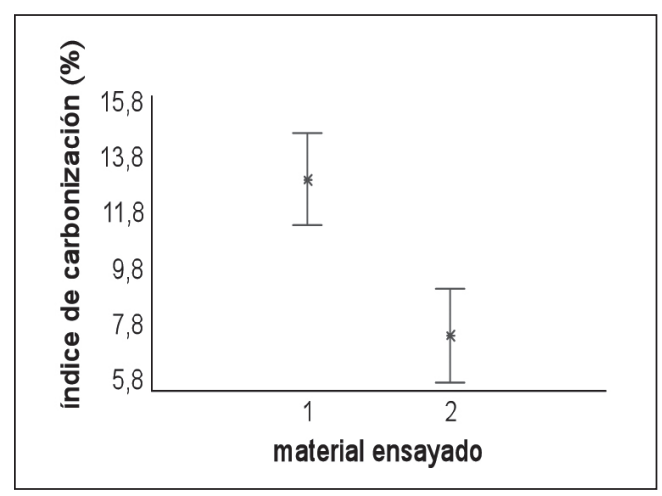

Figura 6. Índices de carbonización entre tableros con y sin retardante 
Se comprobó la diferencia estadística para el índice de carbonización en los tableros de madera con y sin retardante de llamas. Se realizó un análisis de varianza (Tabla 4) para comprobar si existen diferencias significativas entre las medias de los factores.

Tabla 4: Análisis de varianza para índice de carbonización en tableros de madera.

\begin{tabular}{cccccc}
\hline \multicolumn{7}{c}{ Análisis de la Varianza } & & \\
\hline Fuente & Sumas de cuadrados & Gl & Cuadrado Medio & Cociente-F & P-Valor \\
\hline Entre factores & 316,40 & 1 & 316,40 & 11,67 & 0,0015 \\
\hline $\begin{array}{c}\text { Dentro de los } \\
\text { factores }\end{array}$ & 1030,1 & 38 & 27,10 & & \\
\hline Total (Corr.) & 1346,51 & 39 & & & \\
\hline
\end{tabular}

Se determinó que existen diferencia estadísticamente significativa, con un 95\% de confianza, del índice de carbonización entre los tableros de madera con el retardante de llama (2) y los tableros sin recubrimiento (1). Por medio de una análisis de contraste múltiple (Figura 6) se corroboró que los tableros con retardante de llama tuvieron un menor índice de carbonización que los sin retardante de llama.

Al realizar un análisis estadístico entre tableros estructurales OSB y contrachapados con y sin retardante de llamas se comprobaron diferencias estadísticas de pérdida de peso. Se realizó un análisis de varianza (Tabla 5) para comprobar si existían diferencias significativas entre las medias de los factores.

Tabla 5. Análisis de varianza para pérdida de peso en tableros OSB y Contrachapados.

\begin{tabular}{cccccc}
\hline \multicolumn{7}{c}{ Análisis de la Varianza } \\
\hline Fuente & Sumas de cuadrados & Gl. & Cuadrado Medio & Cociente-F & P-Valor \\
\hline Entre factores & 163,42 & 3 & 54,47 & 4,72 & 0,0152 \\
\hline $\begin{array}{c}\text { Dentro de los } \\
\text { factores }\end{array}$ & 184,74 & 16 & 11,54 & & \\
\hline Total (Corr.) & 348,17 & 19 & & & \\
\hline
\end{tabular}

Se determinó que existe diferencia estadísticamente significativa, con un 95\% de confianza, entre la pérdida de peso de los tableros OSB sin retardante de llama (1), OSB con retardante de llama (2), contrachapado sin retardante de llama (3) y contrachapado con retardante de llama (4). Por medio de un análisis de contraste múltiple se corroboró que existen diferencias significativas entre tableros OSB y contrachapados con retardante de llamas (Figura 7). Además, los tableros contrachapados con retardante de llama arrojaron diferencias significativas respecto a los demás.

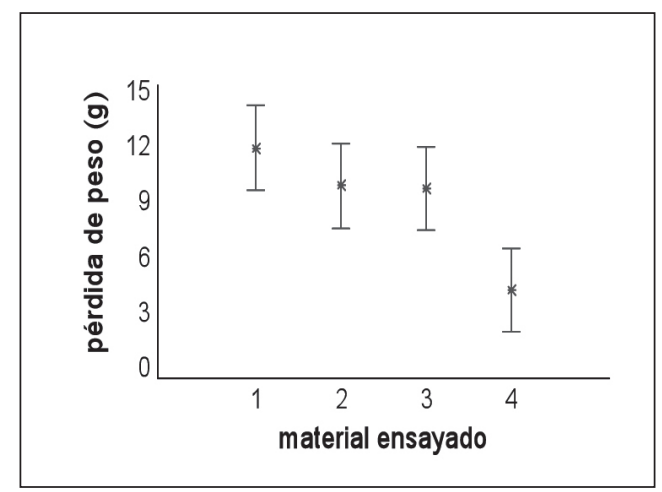

Figura 6. Comparación en pérdida de peso para OSB y Contrachapados

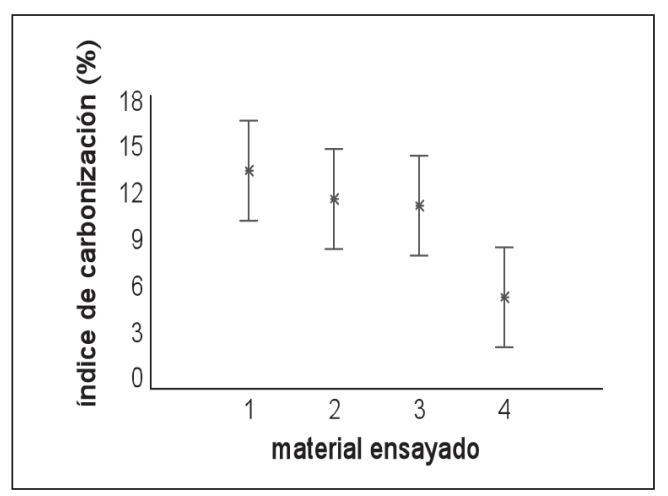

Figura 7. Comparación del índice de carbonización para OSB y Contrachapados 
Se comprobó la diferencia estadística para el índice de carbonización de los tableros OSB y Contrachapado con y sin retardante de llama. Se realizó un análisis de varianza (Tabla 7) para comprobar si existían diferencias significativas entre las medias de los factores.

Tabla 7. Análisis de varianza para índice de carbonización para tableros OSB y Contrachapados.

\begin{tabular}{|c|c|c|c|c|c|}
\hline \multicolumn{7}{|c|}{ Análisis de la Varianza } \\
\hline Fuente & Sumas de cuadrados & Gl & Cuadrado Medio & Cociente-F & P-Valor \\
\hline Entre factores & 188,461 & 3 & 62,8205 & 2,69 & 0,0809 \\
\hline Dentro de los factores & 373,204 & 16 & 23,3252 & & \\
\hline Total (Corr.) & 561,665 & 19 & & & \\
\hline
\end{tabular}

Se determinó que no existe diferencia estadísticamente significativa, con un 95\% de confianza, entre el índice de carbonización (Figura 8) en tableros OSB sin producto protector (1), OSB con retardante de llamas (2), Contrachapado sin producto protector (3) y Contrachapado con retardante de llama (4). Por medio de una análisis de contrate múltiple se corroboró que no existen diferencias significativas entre tableros OSB y Contrachapados con retardante de llamas. Entre los tableros OSB, con y sin retardante, tampoco se registraron diferencias significativas, al igual que entre los tableros Contrachapados.

\section{CONCLUSIONES}

Todos los tableros ensayados mejoraron su comportamiento frente al fuego cuando se les aplicó un retardante de llama.

Se corroboró que los tableros a base de madera y la madera de pino radiata con retardante de llama pueden resistir más en exposición directa de fuego, ya que mejoran las propiedades ignifugas, disminuyendo la pérdida de peso y el índice de carbonización debido al aumento del punto de ignición y disminución del desplazamiento y penetración de la llama.

Al comparar entre tableros, Contrachapado presenta mejor desempeño al comparar con OSB y partículas resulta menos dañado al comparar con MDF frente a la acción directa de la llama. El comportamiento más deficiente se observó en tableros de fibra HB.

\section{BIBLIOGRAFÍA}

Arquicity. 2006. Guia de Ignífugos: Retardantes de fuego para la madera. 2006. [En Línea]. <http:// www.arquicity.com/guia-ignifugos-retardantes.html>

[Consulta: Julio 2008].

ASTM. 1990. Standard test method for Small Scale Evaluation of Fire-Retardant Paints. American Society for Testing and materials. Philadelphia, PA. D 3806- $90^{\mathrm{a}}$

ASTM.1992. Standard test method for heat and visible smoke release rates for materials and products an oxygen consumption calorimeter. American Society for Testing and materials. Philadelphia, PA. (1354-92).

ASTM. 1994. Standard Test Method for Fire Retardancy of Paints (Cabinet Method). Annual Book of ASTM Standards. 6(1): 190-192. American Society for Testing Materials. Philadelphia, PA. D1360$90 \mathrm{a}$ 
Castillo, M. 2003. Protección pasiva contra el fuego. Revista BIT. Edición Especial. Mayo 2003: 57-59.

Chuen-Shii, C.; Sheau-Horng, L.; Chin-I, W. 2009. Preparation and characterization of the intumescent fire retardant coating with a new flame retardant. Advanced Powder Technology 20 (2009) 169-176. Published by Elsevier B.V. on behalf of The Society of Powder Technology Japan.

Decorespacio. 2008. Productos retardantes del fuego. [En línea]. <http://www.decorespacio. com/20080415-productos-retardantes-del-fuego.html>

[Consulta: Julio 2008]

Forest Products Laboratory. 1999. Wood handbook - Wood as an engineering material.

Gen. Tech. Rep. FPL-GTR-113. Madison, WI: U.S. Department of Agriculture, Forest Service, Forest Products Laboratory. 463 p.

Garay, R. ; Poblete, H. ; Karsulovic , J.T. 2009. Evaluation of oriented strandboard and plywood subjected to severe relative humidity and temperature conditions. Forest Prod. J. 59(3):84-90.

Garay, R.; Ahumada, I. 2008. Comparación de propiedades de suelos laminados. Boletín de Información Técnica 252: 26:29.

Garay, R. 2007. Impregnantes tipo lasur para la protección de la madera y tableros. Agro-Ciencia 23(1): 25-36.

Garay, R. 2003. Informe Técnico Paneles Arauco - Planta Trupan - Molduras. 18 p.

Garay, R.; González, J.; Vaccarezza, F. 1996. Evaluación técnica de aplicación de retardantes de fuego a tableros MDF. Actas VIII Reunión de Investigación de Productos Forestales. Universidad de Chile. 470-483.

Harada, T.; Uesugi, S.; Taniuchi, H. 2003. Evaluation of fire-retardant wood treated with polyphosphatic carbamate using a cone calorimeter. Forest Products Journal 53: 81-85

Hashim, R. O.; Sulaiman, R.N.; Kumar, P.F.; Tamyez, R.J.; Murphy, Z. A. 2009. Physical and mechanical properties of flame retardant urea formaldehyde medium density fiberboard. Journal of Materials Processing Technology 209: 635-640.

Infante, R. 2008. Sistemas resistentes al fuego y al calor, intumescentes, retardantes e ignífugos. Ceresita S. A. Santiago, Chile. 33-35.

INFOR. 1999. Reaction to fire testing of wood products. AB Trätek, Swedish Institute for Wood Technology Research Laboratory, Stockholm No. A12100/991102. 10 p.

INN. 1997. Prevención de incendio en edificios. Ensayo de resistencia al fuego. Parte 1: Elementos de construcción en general. NCh 935/1 - of 97.

INN. 1986. Prevención de incendio en edificios. Pinturas. Determinación del Retardo al fuego. NCh 1974 of 86.

Jun-wei, G.; Guang-cheng, Z.; Shan-lai, D.; Qiu-yu, Z.; Jie, K. 2007. Study on preparation and fireretardant mechanism analysis of intumescent flame-retardant coatings. Surface \& Coatings Technology 201: 7835-7841 
Lebow, S.; Winandy, J. 1998. The role of grade and thickness in the degradation of fire-retardanttreated plywood. Forest Products Journal 48(6): 88-94

Lp Chile. 2008. Manual práctico de Construcción Lp. [En Línea] htttp://www.lpchile.cl/manualLP/ anexos/10_ANEXO_ENSAYOS\%20FIR333-340.pdf. [Consulta: Julio 2008].

MINVU. 2004. Nuevo Listado Oficial de comportamiento al fuego de elementos y componentes de la construcción. Título V: De las Tablas y Ensayos Informativos de Cálculo del Comportamiento al Fuego de los Elementos y Componentes de la Construcción. Santiago, Chile. 219-221.

Neira, C. 2001. Innovación En Métodos Constructivos. Novedades e Innovaciones. Revista Técnica de la Construcción (BIT): 21-24.

Peraza, F. 1998. Tableros de viruta OSB. Wood based panels. Revista AITIM Julio - Agosto. 15p.

Ramírez, G.I.; Di Pace, A. 2002. Comportamiento al fuego de revestimientos de madera. Materiales, Desarrollo tecnológico y precompetitivo. $4^{\circ}$ Jornadas de Desarrollo e Innovación. [En línea]. 2 págs. http://www4.inti.gov.ar/GD/4jornadas2002/pdf/cecon-016.pdf. [Consulta: Julio 2008]

QUIMINET. 2006. Los retardantes de fuego. 2006. [en línea]. <http://www.quiminet.com.mx/ar1/ar _4\%2514\%2501\%2512\%251B\%2510\%2560\%2501.htm>[Consulta Julio 2008] 